\title{
INFLUÊNCIA DOS PARÂMETROS DE PROCESSAMENTO NAS PROPRIEDADES MECÂNICAS DE COMPÓSITOS DE MATRIZ POLIESTER REFORCCADOS COM FIBRAS DE CURAUÁ DE ALTA PERFORMANCE*
}

\section{Resumo}

Noan Tonini Simonassi ${ }^{1}$ Fabio de Oliveira Braga ${ }^{2}$ Sergio Neves Monteiro ${ }^{3}$

As fibras naturais extraídas das folhas do curauá estão entre as mais fortes fibras naturais utilizadas como reforço de compósitos poliméricos para aplicações em engenharia. Tais compósitos, até agora, são limitados em relação às propriedades mecânicas e energia de impacto. Assim o objetivo do presente trabalho é de investigar a possibilidade da melhora da resistência à tração bem como a energia de impacto dos compósitos de matriz poliéster reforçados com fibras de curauá com variados parâmetros de processamento. Os parâmetros observados foram a quantidade de fibras com diâmetro fino e pressão aplicada durante o processamento. Utilizando $60 \%$ de fração volumétrica de fibras finas (diâmetro médio variando de 50 à $80 \mu \mathrm{m}$ ) contínuas e alinhadas foi possível se obter resistência à tração superior $200 \mathrm{MPa}$. Ainda, aplicando-se $30 \mathrm{MPa}$ de pressão durante a cura foram obtidos mais de $270 \mathrm{MPa}$ de resistência a tração. Já para resistência a impacto Izod, uma resistência de $340 \mathrm{~J} / \mathrm{m}$ foi obtido pelos corpos de prova com entalhe feito em sentido perpendicular ao alinhamento das fibras.

Palavras-chave: Fibras naturais, Fibras de curauá, Compósitos, Propriedades Mecânicas.

\section{INFLUENCE OF PROCESSING PARAMETERS IN THE MECHANICAL PROPERTIES FOR HIGH PERFORMANCE POLIÉSTER COMPOSITES REINFORCED WITH CURAUA FIBERS}

\section{Abstract}

The lignocellulosic fiber extracted from the curaua plant leafs is among the strongest natural fibers used as polymer composite reinforcement for engineering application. Such composites, so far, are limited in mechanical strength and impact energy. Therefore, the objective of the present work was to investigate the possibility of improving the tensile strength as well as the impact energy of curaua fiber reinforced polyester composites by varying process parameters. These parameters were the amount of fibers with thinner diameters and applied pressure during composite preparation. Using $60 \mathrm{vol} . \%$ of thinner, 50 to $80 \mu \mathrm{m}$, continuous and aligned curaua fibers it was possible to obtain over $200 \mathrm{MPa}$ of tensile strength. Moreover, by applying $30 \mathrm{MPa}$ of pressure during curing, more than $270 \mathrm{MPa}$ was obtained. As for lzod impact resistance, a toughness of over $340 \mathrm{~J} / \mathrm{m}$ was obtained for specimens with notchmachined perpendicular to the fiber alignment.

Keywords: Natural Fibers, Curaua Fibers, Composites, Mechanical Properties.

Instituto Militar de Engenharia, Doutorando em Ciência dos Materiais - Praça General Tibúrcio, 80, 22.290-270, Praia Vermelha, Urca, Rio de Janeiro - RJ, Brasil.

2 Instituto Militar de Engenharia, Doutorando em Ciência dos Materiais - Praça General Tibúrcio, 80, 22.290-270, Praia Vermelha, Urca, Rio de Janeiro - RJ, Brasil.

3 Instituto Militar de Engenharia, Professor, Ph.D. em Engenharia e Ciências dos Materiais - Praça General Tibúrcio, 80, 22.290-270, Praia Vermelha, Urca, Rio de Janeiro - RJ, Brasil 


\section{INTRODUÇÃO}

Fibras lignocelulósicas são materiais naturais resistentes que vem sendo utilizadas desde tempos antigos como matérias primas para a produção de produtos manufaturais como tecidos, cordas, velas de navegação, tela de pintura dentre outras [1-4]. No último século, contudo, houve a perda de mercado destas que foram, em parte, substituídas pelas fibras sintéticas [5]. Mais recentemente, entretanto, as fibras naturais vem ganhando significância por estas serem renováveis, e diminuírem, durante a confecção dos compósitos, o consumo de energia total, que está associado, basicamente, a queima de combustíveis fósseis e reduzirem a emissão de $\mathrm{CO}_{2}$ e outros gazes de efeito estufa que são responsáveis pelo fenômeno de aquecimento global [4-10].

A importância das fibras naturais e seus compósitos não estão restritas às questões ambientais. Sua alta resistência à tração e elevado Módulo de Elasticidade, baixo peso e baixo custo bem como boas propriedades térmicas e isolamento acústico são outras características interessantes que tornam estas promissoras para aplicação em várias indústrias [11-12].

Entre as fibras naturais, as fibras naturais extraídas das folhas da planta Ananas Erectifolius, popularmente conhecida como curauá, estão sendo investigadas, devido a sua alta resistência à tração [13] e elevado Módulo de Elasticidade [13-14] e tem sido, portanto, consideradas para várias aplicações dentre as quais, as mais promissoras são para peças automobilísticas [15-19]. Para estas fibras, valores de $3000 \mathrm{MPa}$ de resistência a tração e Módulo de Young de $80 \mathrm{GPa}$ são reportados na literatura [10-15].

Estudos anteriores demonstraram que propriedades, tais quais densidade e Módulo de Elasticidade das fibras naturais lignocelulósicas tendem a ser inversamente proporcional em relação ao seu diâmetro [2, 10, 16, 22]. É, então, esperado que os compósitos com melhor desempenho podem ser obtidos com a seleção das fibras finas em relação as fibras com diâmetro aleatório, ou seja, quando não há a seleção destas. Além disso, parâmetros como pressão durante a moldagem e orientação da fibra também interferem no comportamento mecânico do compósito. Assim, o objetivo do presente trabalho é avaliar o desempenho de compósitos de matriz poliéster reforçados com elevadas frações volumétricas de fibra de curauá submetidos a ensaios de tração bem como a influência da orientação das fibras na resistência a impacto deste material. As propriedades analisadas foram comparadas com os de compósitos confeccionados de maneia convencional, com fibras de diâmetro aleatório e sem alguma orientação preferencial.

\section{MATERIAIS E MÉTODOS}

As fibras de curauá utilizadas neste trabalho são fornecidas pela empresa Pematec Triangel na forma de feixes conforme mostrado na figura 1. O diâmetro destas fibras variam entre 0,05 e 0,23 $\mathrm{mm}$ com um diâmetro médio de 0,094 $\mathrm{mm}$ conforme pode ser observado no histograma também presente na figura 1 


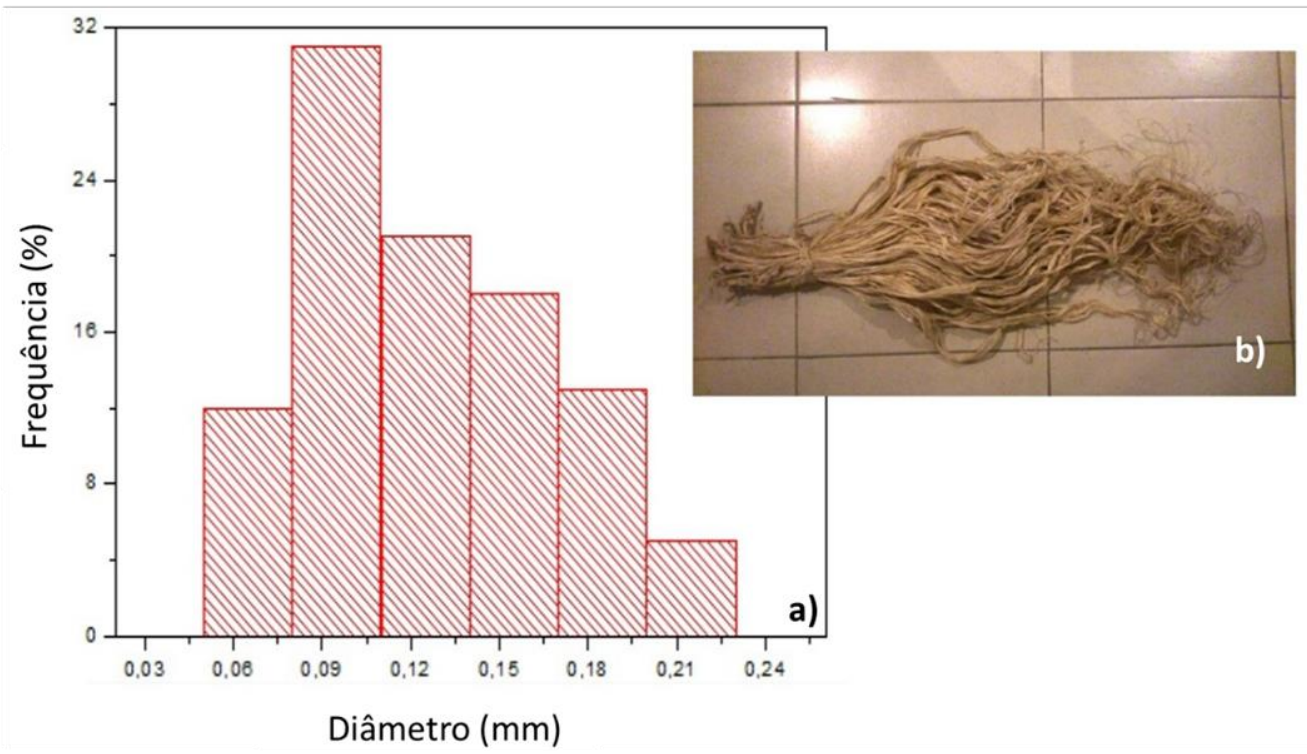

Figura 1: Histograma da distribuição de diâmetro das fibras de curauá (a) e o feixe conforme recebido (b).

Para a confecção do compósito, uma resina poliéster insaturada foi utilizada como matriz misturada com o endurecedor metil etil cetona na proporção em massa de $2 \%$ de endurecedor para resina fornecido pela empresa Resinpoxy. As fibras de curauá utilizadas como reforço foram manualmente limpas, cortadas, secas por 24 horas à $60 \stackrel{\circ}{\circ}$ em estufa e postas junto com a resina. A mistura foi deixada em um molde metálico para que ocorresse a cura do polímero durante 24 horas.

Os corpos de prova de tração foram confeccionados em um molde projetado especialmente para que as amostras, ao serem retiradas, possuíssem as dimensões especificadas na norma ASTM D638 [23], variando se as frações volumétricas de fibra utilizadas (30, 40, 50,60, 70, 80 e 90\%). Após testes preliminares, a fração de fibras que apresentou melhor desempenho foi escolhida como padrão de comparação e os demais corpos de prova foram confeccionados com base neste valor. Fibras finas, com diâmetro médios selecionados entre 0,05 à $0,08 \mathrm{~mm}$, foram utilizadas para a preparação destes compósitos. Os testes de tração foram conduzidas até a fratura em uma máquina Instron modelo 3365 disponível no Laboratório de Ensaios Não Destrutivos Corrosão e Soldagem (LNDC) da Universidade Federal do Rio de Janeiro (UFRJ) utilizando-se célula de carga de 30 $\mathrm{kN}$ em uma taxa de deformação de 0,75 $\mathrm{mm} / \mathrm{min}$. Os tipos de compósitos ensaiados em tração foram: Corpos de prova de tração com fibras finas e sem pressão durante a cura (FFS/P); Corpos de prova de tração com fibras finas e com pressão (30 MPa) durante a cura (FFC/P); Corpos de prova de tração com fibras de diâmetro aleatório e com pressão (30 MPa) durante a cura (FDA). Os resultados obtidos foram sujeitos a análise estatística de Weibull.

Os ensaios de impacto Izod foram conduzidos em um pêndulo Pantec $\mathrm{CH} / \mathrm{IZ}-25 \mathrm{~J}$ utilizando-se um martelo de $22 \mathrm{~J}$, em temperatura controlada de $23 \stackrel{\circ}{\circ}$. As amostras retangulares $(65 \times 12 \times 12 \mathrm{~mm})$ foram cortadas de uma placa retangular $(120 \times 150 \times$ $12 \mathrm{~mm}$ ) de compósito reforçado com 30 \% em fração volumétrica de fibra de curauá, 
produzida em um molde metálico retangular sob pressão de $3 \mathrm{MPa}$. As dimensões finais dos corpos de prova são as especificadas pela norma ASTM D256 [24]. Por fim, um entalhe com $45 \pm 1^{\circ}$ de abertura e $0,25 \pm 0,05 \mathrm{~mm}$ de raio de curvatura foi feito em cada amostra segundo as recomendações da norma. Nesta análise levouse consideração a orientação da fibra em relação ao entalhe e três tipos de corpos de prova foram confeccionados: De resina pura (RP); De entalhe em sentido paralelo à orientação das fibras $(\mathrm{E} / / \mathrm{F})$; De entalhe perpendicular à orientação das fibras $(E \perp F)$.

A superfície de fratura das amostras foi observada em um Microscópio Eletrônico de Varredura (MEV) Jeol modelo JSM-5800LV afim de se verificar os mecanismos de fratura dos compósitos.

\section{RESULTADOS E DISCUSSÃO}

\subsection{Resultados de Tração}

A figura 2 mostra as curvas com os resultados preliminares de resistência a tração dos compósitos em função da quantidade de fibra para corpos de prova feitos com fibras de diâmetro aleatórios e fibras finas. Para os compósitos de fibras finas, houve uma tendência de aumento de resistência com o incremento de fração volumétrica de fibras até $60 \%$, alcançando $210 \pm 1 \mathrm{MPa}$, e um decréscimo com maiores frações volumétricas adicionadas. Para os compósitos de fibras de diâmetros aleatórios 0 mesmo comportamento foi observado onde estes alcançaram o maior valor de resistência a tração (157 $\pm 12 \mathrm{MPa}$ ) em $80 \%$ de fração volumétrica, diferentemente dos compósitos reforçados com fibras finas.

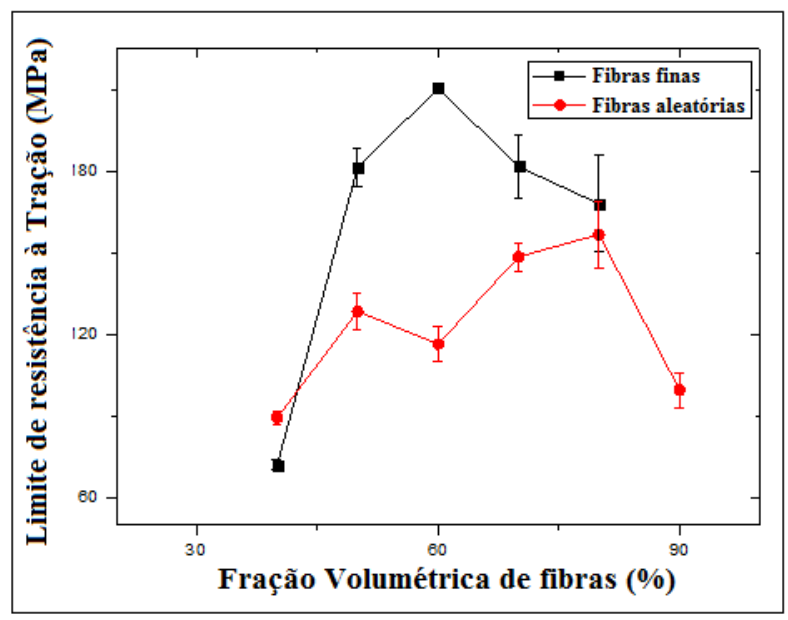

Figura 2: Resistência a tração dos compósitos reforçados com fibras de diâmetro aleatório e fibras finas.

Como pode ser observado na figura 2, de maneira geral, os compósitos reforçados com fibras finas são mais resistentes do que os reforçados com fibras não selecionadas. Esse comportamento é esperado, já que as fibras finas são mais resistentes como observado em trabalhos anteriores [22]. Ainda, as fibras finas são mais densas [22], fazendo com que, para a mesma fração volumétrica, uma maior quantidade de fibras seja utilizada para reforçar o compósito. Assim, os compósitos 
reforçados com esse tipo de fibra possui uma distribuição mais homogênea, aumentando assim a resistência.

O valor de $60 \%$ fração volumétrica de fibras foi o escolhido como parâmetro para os próximos ensaios já que foram os melhores resultados obtidos. A figura 3 mostra a distribuição de Weibull para os compósitos FFS/P, FFC/P e FDA. Com a aplicação de $30 \mathrm{MPa}$ de pressão durante a cura, os compósitos apresentaram aumento de resistência à tração de $157 \pm 26 \mathrm{MPa}$ (resultados preliminares) para $274 \pm 9 \mathrm{MPa}$ (74\% de aumento) para os compósitos de fibras finas. Além disso, quando comparados os compósitos FDA e FFC/P ocorre um aumento de 188,1 $\pm 27 \mathrm{MPa}$ para $274 \pm 9 \mathrm{MPa}$ (45\% de aumento).

A análise de Weibull fornece outras informações importantes. Como pode ser observado, ocorre uma melhora, não só na resistência média, mas também na distribuição estatística da resistência. Tanto a seleção das fibras finas quanto o uso de pressão durante a cura, acarretam em uma diminuição na dispersão estatística dos resultados. Isso pode ser numericamente quantificado pelo parâmetro $\beta$, que é 3 vezes maior para o caso dos compósitos de FFC/P em relação aos compósitos FFS/P (30,7 e 9,9, respectivamente) e 5,6 vezes maior quando comparado com os compósitos FDA (30,7 e 5,5, respectivamente).

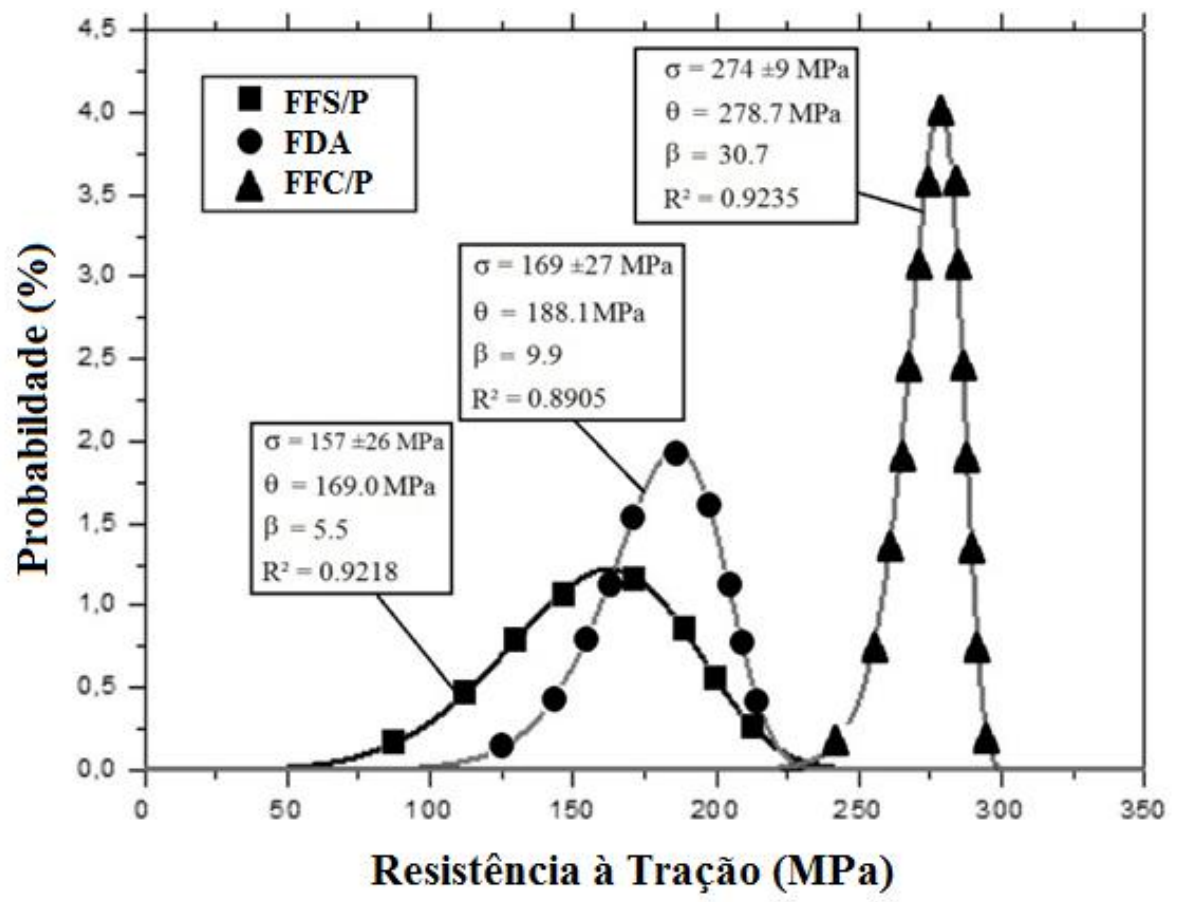

Figura 3: Gráfico de função de densidade de probabilidade de Weibull para os diferentes compósitos.

A superfície de fratura dos compósitos FFC/P foram analisadas e a micrografias é apresentada na figura 4. O descolamento observado entre a fibra e a matriz pode ser considerada como indicativo de fraca interface entre as fases, como são esperados para os compósitos reforçados com fibras naturais lignocelulósicas de curauá [8]. Contudo, o descolamento não foi completo, o que pode ser entendido 
como uma melhora na resistência da interface proporcionado pela preção exercida pelo molde durante a cura do polímero.

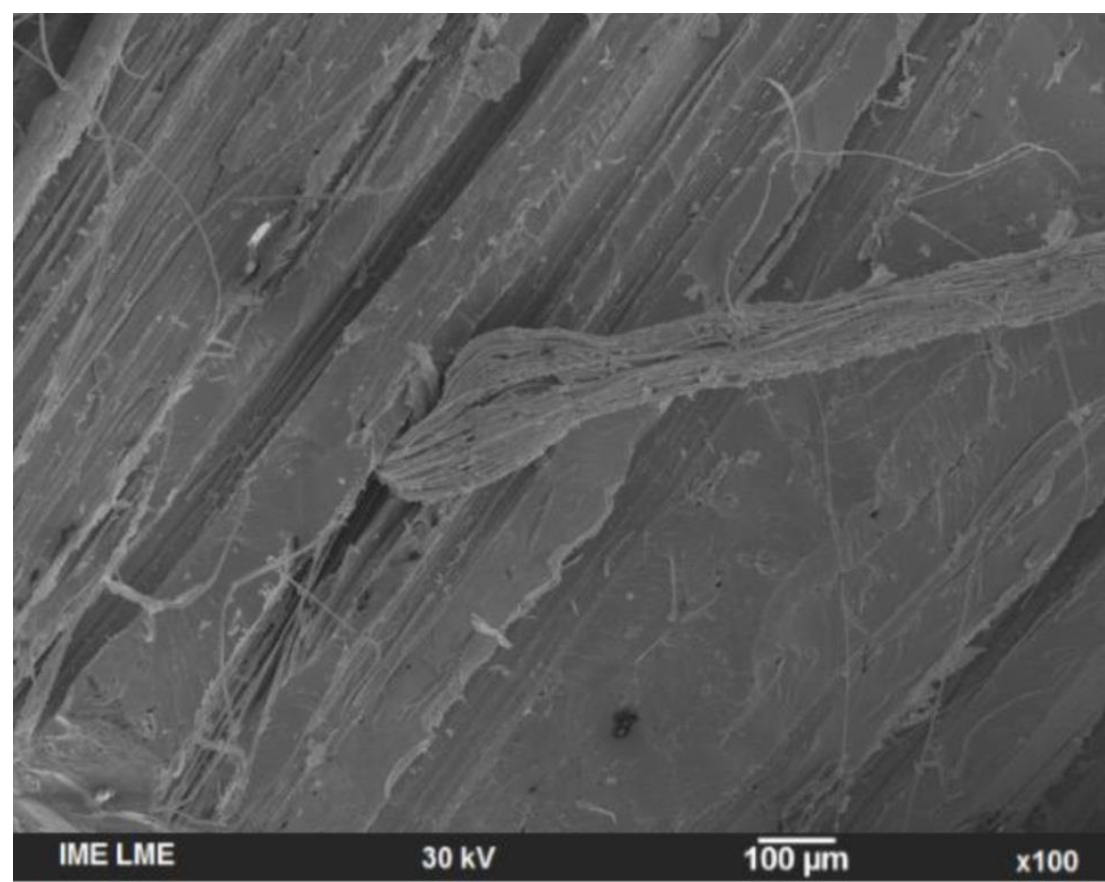

Figura 4: Micrografia por MEV da superfície de fratura mostrando o descolamento entre fibra e matriz.

\subsection{Ensaios de Impacto Izod.}

A figura 5 mostra a comparação de resistência ao impacto Izod entre os dois tipos de orientação de fibras $(E / / F$ e $E \perp F)$ dos compósitos reforçados com fibras naturais de curauá e a resina pura (RP). A configuração $E / / F$ proporciona uma pequena resistência ao impacto em relação à $\mathrm{RP}$, aumentando levemente a resistência ao entalhe de 16,2 $\pm 1,7$ para 19,8 $\pm 1,5 \mathrm{~J} / \mathrm{m}$ e resistência ao impacto de 1,8 $\pm 0,1$ para $2,2 \pm 0,1 \mathrm{~kJ} / \mathrm{m}^{2}$. Contudo, os compósitos $E \perp F$ apresentaram incremento significativo na resistência, apresentando $341,7 \pm 0,5 \mathrm{~J} / \mathrm{m}$ de resistência ao entalhe, e 36,6 $\pm 4,8$ $\mathrm{kJ} / \mathrm{m}^{2}$ de resistência ao impacto (aumento de 20 vezes em comparação com a resina pura. Em um trabalho recente, Braga et al., 2017 [25] estudou a fibra de curauá em uma configuração em reforço de resina epóxi em ambas as direções, utilizando-se uma manta feita com a fibra. Nesta configuração, foi observado um aumento de resistência de cerca de 10 vezes. Os resultados encontrados no presente trabalho confirma que fibras contínuas e alinhadas como reforço em compósitos são suscetíveis à fratura quando submetidas a forças de impacto de origem desconhecidas. 

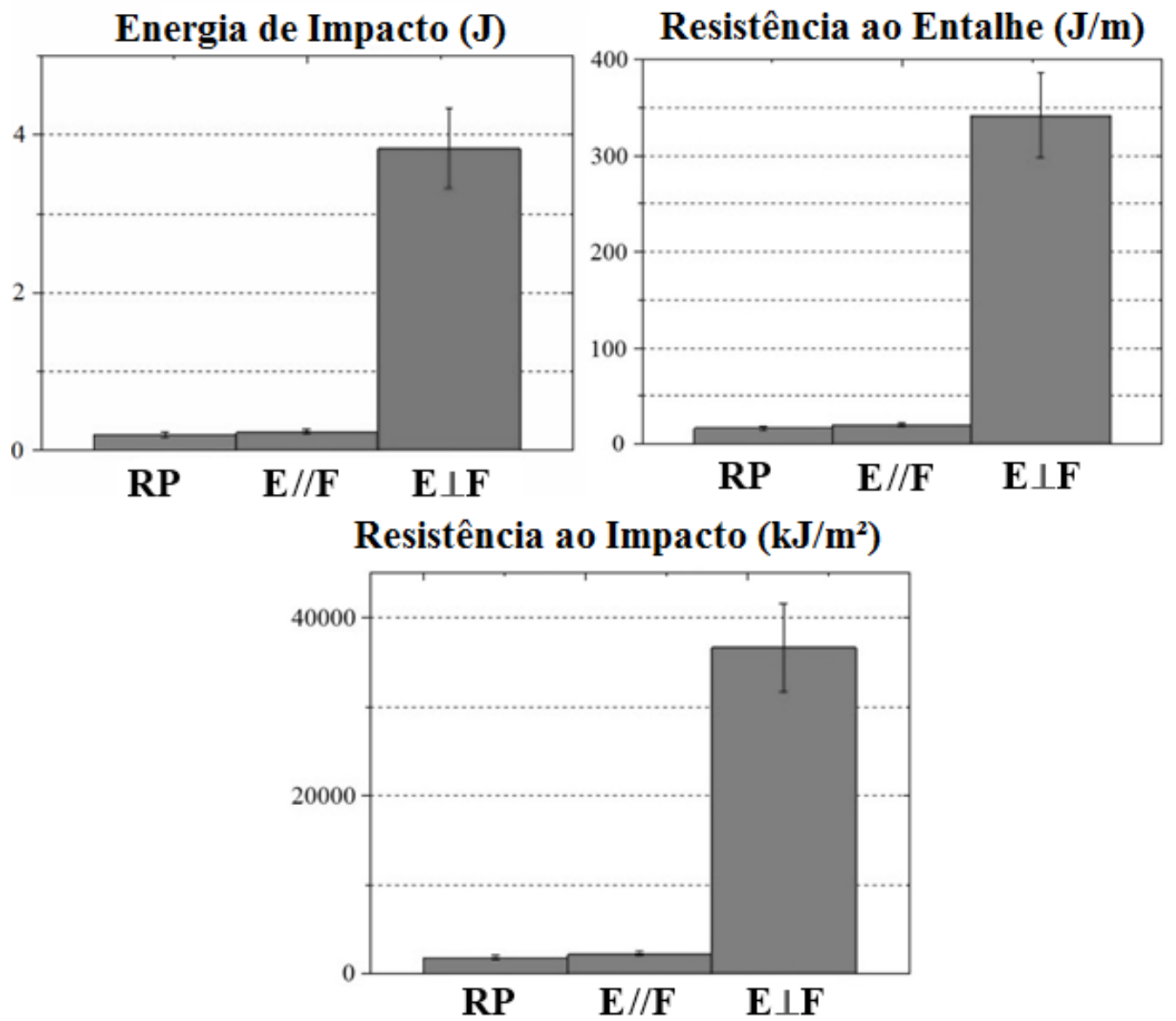

Figura 5: Valores de resistência ao impacto para $R P, E / / F$ e $E \perp F$.

A figura 6 mostra as amostras fraturadas. Pode ser observado que para a RP e E//F os corpos de prova apresentaram fratura facilitada em relação aos compósitos $E \perp F$, que não apresentaram fratura total.

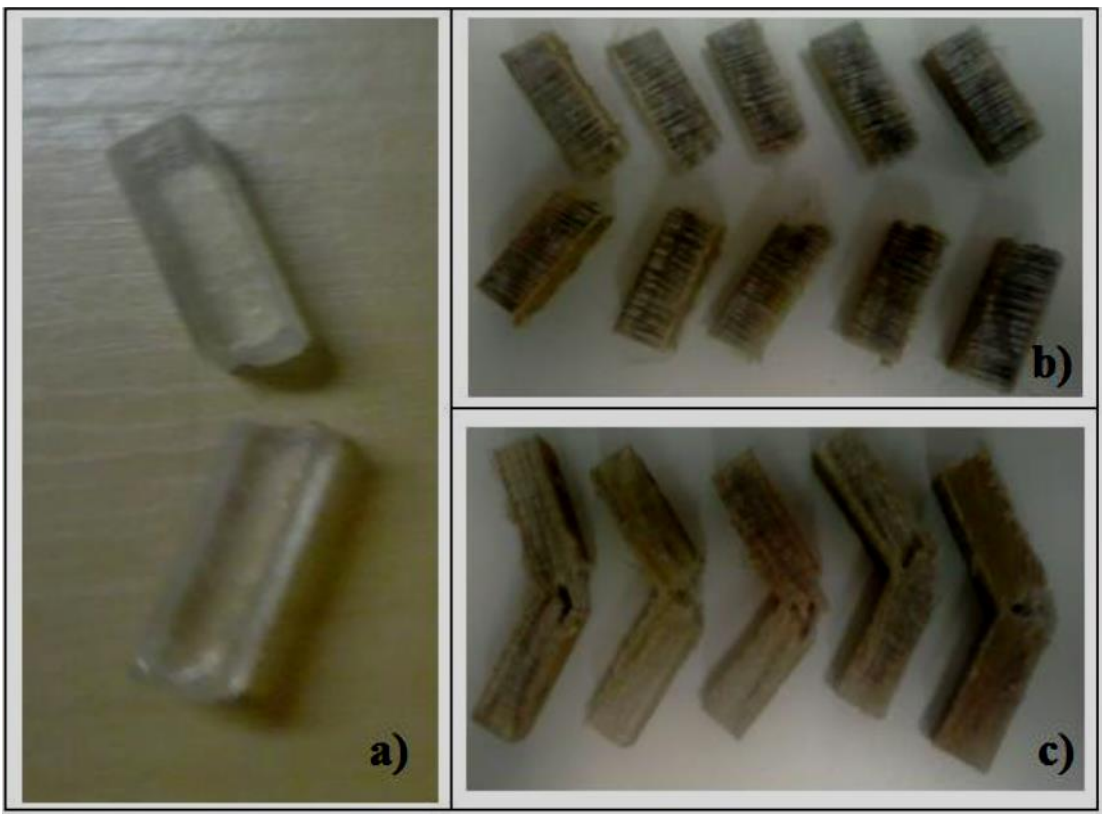

Figura 6: Aspecto geral da fratura dos corpos de prova de impacto. 
A micrografia em MEV da superfície de fratura de um compósito $E \perp F$ (figura 7) mostra que o compósito obtém um reforço efetivo das fibras e as trincas geradas no entalhe devem mudar de direção para continuar a se propagar até romper as fibras em algum ponto. $O$ resultado deste comportamento é que quando as fibras são postas no sentido $E \perp F$ aumentam significativamente a resistência do material em comparação com os outros tipos de compósitos estudados.

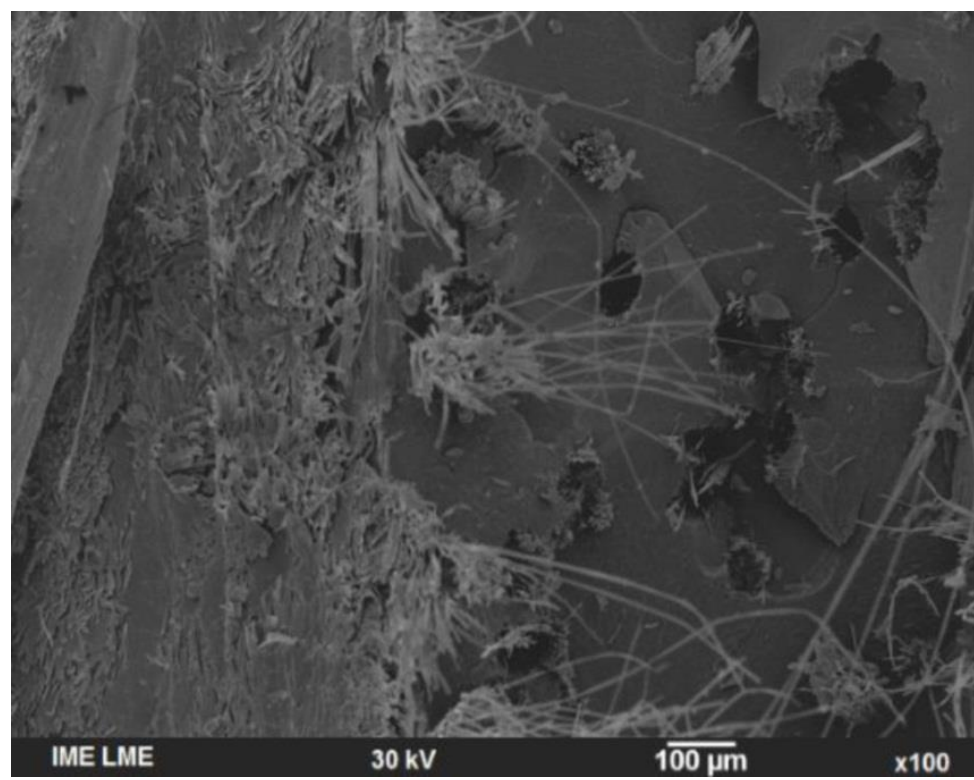

Figura 7: Imagem de MEV mostrando a fratura de impacto de compósitos reforçados com fibras de curauá do tipo $\mathrm{E} \perp \mathrm{F}$.

\section{CONCLUSÕES}

As propriedades de resistência a tração e ao impacto de compósitos de matriz poliéster reforçados com fibras naturais foram avaliadas.

- A composição que apresentou o melhor desempenho de resistência a tração foi o de $60 \%$ de fração volumétricas de fibras.

- Fibras finas (com diâmetro entre 0,05 e 0,08 mm) foram selecionadas e seus compósitos apresentaram resultados $62 \%$ superiores $274 \pm 9 \mathrm{MPa}$ ) em relação aos compósitos com fibras de diâmetros aleatórios (169 $\pm 27 \mathrm{MPa}$ ).

- Os compósitos confeccionados com $30 \mathrm{MPa}$ de pressão durante a cura do polímero apresentaram um desempenho $74 \%$ melhor do que os compósitos confeccionados sem pressão ( $157 \pm 26 \mathrm{MPa})$.

- A orientação paralela ao entalhe das fibras proporciona muito pouco aumento de resistência ao impacto em comparação à resina pura. Contudo, o reforço colocado de maneira perpendicular ao entalhe melhora a resistência ao entalhe mais de 20 vezes em comparação ao poliéster puro, alcançando $341,7 \pm 0,5$ $\mathrm{J} / \mathrm{m}$.

\section{AGRADECIMENTOS}

Os autores gostariam de agradecer o suporte para este trabalho às agências de fomento CNPq, CAPES e FAPERJ, bem como ao LNDC/UFRJ pelos ensaios de tração. 


\section{REFERÊNCIAS}

1. Bledzki AK, Gassan J. Composites reinforced with cellulose based fibers. Prog. Polym. Sci., 24, 221. 1999.

2. Crocker J. Thermogravimetric Stability Behavior of Less Common Lignocellulosic Fibers - a Review. Mater. Technol., 23, 3, 174. 2008.

3. Thomas, S.; Paul, S.A.; Pothan, L.A.; Deepa, B. in Natural Fibres: Structure, Properties and Applications, ed. Kalia, S.; Kaith, B.S.; Kaur, I. Berlin: Springer, p. 24. 2011.

4. Eichorn SJ, Dufresne A, Aranguren M, Marcovich NE, Capadona JR, Rowan SJ, Weder C, Thielemans W, Roman M, Renneckar S, Gindl W, Veigel S, Keckes J, Yano $\mathrm{H}$, Abe K, Nogi M, Nakagaito AN, Mangalam A, Simonsen J, Benight AS, Bismarck A, Berlung AL, Peijs T. Review: current international research into cellulose nanofibres and nanocomposites J. Mater. Sci., 45, 1. 2010.

5. Monteiro SN, Lopes FPD, Ferreira AS, Nascimento DCO. Natural-fiber polymermatrix composites: Cheaper, tougher, and environmentally friendly. JOM, 61, 17. 2009.

6. Faruk O, Bledzki AK, Fink H, Sain M. Biocomposites Reinforced with Natural Fibers: 2000-2010. Prog. Polym. Sci., 37, 1552. 2012.

7. Mohanty AK, Misra M, Drzal LT. Sustainable Bio-Composites from Renewable Resources: Opportunities and Challenges in the Green Materials World. J. Polym. Environ. 10, 19. 2002.

8. Netravali AN, Chabba S. Composites get greener. Mater Today., 6, 22. 2003.

9. Satyanarayana KG, Arizaga GGC, Wypych F. Biodegradable composites based on lignocellulosic fibers - An overview. Progress Polym Sci., 34, 982. 2009.

10. Monteiro SN, Lopes FPD, Barbosa AP, Bevitori AB, Silva ILA, Costa LL. Natural Lignocellulosic Fibers as Engineering Materials - An Overview Met. Mat. Trans. A, 2011, 42, 2963. 2011.

11. Faruk O, Bledzki AK, Fink H. Sain, M. Progress report on natural fibre reinforced composites. Macromol. Mater. Eng., 299, 9. 2014.

12. Satyanarayana KG, Sukumaran K, Kulkarni AG, Pillai SGK, Rohatgi PK. Composites Compos. Part A. 17, 4, 329. 1986.

13. Oliveira FH, Helfer AL, Amico SC. Mechanical Behavior of Unidirectional Curaua Fiber and Glass Fiber Composites. Macromol. Symp., 319, 83. 2012.

14. Almeida Júnior JH, Ornaghi Júnior HL, Amico SC, Amado FDR. Study of hybrid intralaminate curaua/glass composites. Mater. Design, 42, 111. 2012.

15. Zah R, Hischier R, Leão AL, Braun I. Curauá fibers in the automobile industry - a sustainability assessment. J. Clean. Prod. 15, 1032. 2007.

16. Tomczak F, Satyanarayana KG, Sydenstricker THD. Studies on lignocellulosic fibers of Brazil: Part III - Morphology and properties of Brazilian curauá fibers. Compos. Pt. A, 38, 2227. 2007.

17. Spinacé MAS, Lambert CS, Fermoselli KKG, De Paoli M. Characterization of lignocellulosic curaua fibres. Carbohydr. Polym. 77, 47. 2009.

18. Castro DO, Ruvolo-Filho A, Frollini E. Materials prepared from biopolyethylene and curaua fibers: Composites from biomass Polym. Test., 31, 880. 2012.

19. Angrizani CCA, Cioffi MOH, Zatteri AJ, Amico SC. Analysis of curaua/glass hybrid interlayer laminates. J. Reinf. Plast. Compos., 33, 5, 472. 2014. 
20. Monteiro SN, Lopes FPD, Nascimento DCO, Ferreira AS, Satyanarayana KG. Processing and properties of continuous and aligned curaua fibers incorporated polyester composites. J. Mater. Res. Technol., 2, 1, 2. 2013.

21. Satyanarayana KG, Guimarães JL, Wypych F. Compos. Pt. A, 38, 1694. 2007.

22. Monteiro SN, Satyanarayana KG, Ferreira AS, Nascimento DCO, Lopes FPD, Silva ILA, Bevitori AB, Inácio WP, Bravo Neto J, Portela TG. Selection of high strength natural fibers. Matéria, 15, 488. 2011.

23. ASTM Designation D638, Standard Method for Tensile Properties of Plastics, West Conshohocken: 2015.

24. ASTM Designation D256, Standard Test Methods for Determining the Izod Pendulum Impact Resistance of Plastics. West Conshohocken, October 2015.

25. Braga FO, Simonassi NT, Cabral AC, Monteiro SN, Assis FS. Tensile and Impact Properties of Two Fiber Configurations for Curaua Reinforced Composites. in Proceedings of the 3rd Panamerican Materials Congress, ed. Meyers MA, et al. (San Diego, CA: The Minerals, Metals and Materials Society, p. 429. 2017. 